\title{
The Human Dorsal Premotor Cortex Generates On-Line Error Corrections during Sensorimotor Adaptation
}

\author{
Ji-Hang Lee ${ }^{1,2}$ and Paul van Donkelaar ${ }^{3,4}$ \\ ${ }^{1}$ Behavioral Brian Sciences, School of Psychology, University of Birmingham, Birmingham B15 2TT, United Kingdom, ${ }^{2}$ School of Sports Science, \\ Sungkyunkwan University, Seoul 135-230, Korea, and ${ }^{3}$ Department of Human Physiology and ${ }^{4}$ Institute of Neuroscience, University of Oregon, Eugene, \\ Oregon 97403-1240
}

\begin{abstract}
A number of different sites in the human brain have been shown to play a role in sensorimotor adaptation. However, the specific role played by each of these structures in the learning process is poorly understood. In the present study, the contribution of the dorsal aspect of the premotor cortex was examined by disrupting activity at this site using transcranial magnetic stimulation (TMS) while subjects wearing prism goggles pointed at visual targets. This manipulation slowed down the rate of adaptation when vision of the hand was available throughout the movement and reduced the presence of on-line trajectory corrections. This was accompanied by a reduced shift in the felt position of the arm. In contrast, TMS did not cause any alteration in the performance of this task when vision of the hand was available only at the end of the movement. Thus, we infer from this pattern of results that the human dorsal premotor cortex contributes to the generation of the visually based on-line error corrections that are responsible for the remapping of arm position sense underlying sensorimotor adaptation.
\end{abstract}

Key words: sensorimotor adaptation; premotor cortex; TMS; learning; human; training

\section{Introduction}

Prism adaptation is a simple form of learning in which one must compensate for discordance between vision and information related to proprioception and motor commands. On initially donning prism goggles, pointing movements are inaccurate, but with repeated trials, participants adapt to the altered visual input and are able to generate accurate responses. Removing the goggles leads to an aftereffect in which the reaching responses are inaccurate in the direction opposite to that observed during the exposure period.

In order for learning to occur during prism adaptation, two things must occur. First, the subject must determine that an error has occurred and correct the error. It has been demonstrated that the manner in which most error corrections occur is dependent on the nature of visual feedback that is available. In particular, when vision of the hand and target are available throughout the movement, the errors can be corrected "on-line". In contrast, if vision is available only at the end of the movement, the errors can only be corrected in a trial-to-trial manner. The second step during adaptation is to remap the visual representation of space and/or the felt position of the arm to remove the conflict induced by the perturbation. It has been demonstrated that the proprioceptive system undergoes the greatest remapping when full vision

Received Sept. 14, 2005; revised Jan. 5, 2006; accepted Jan. 30, 2006.

This work was supported by a grant from the National Science Foundation (BCS-0121916) awarded to P.v.D. We thank the Chris Miall, Paul Dassonville, and the two reviewers for comments on a previous version of this manuscript.

Correspondence should be address to Dr. Paul van Donkelaar, Department of Human Physiology, University of

Oregon, 122C Esslinger Hall, Eugene, 0R 97403-1240. E-mail: paulvd@uoregon.edu.

DOI:10.1523/JNEUROSCI.3898-05.2006

Copyright $\odot 2006$ Society for Neuroscience $\quad 0270-6474 / 06 / 263330-05 \$ 15.00 / 0$ is available, whereas the visual system undergoes the greatest remapping when only terminal vision is available (Redding and Wallace, 1988, 1996, 1997, 2000). Together, this implies that online error corrections lead to a remapping of the felt position of the arm, whereas trial-to-trial error corrections lead to a remapping of the visual representation of space.

Although prism adaptation has been thoroughly described behaviorally, the neural substrate underlying this form of learning is poorly understood. The premotor cortex is a site that very likely contributes to the alterations in motor output induced by wearing prism goggles. Cells in the dorsal premotor cortex (PMd) in particular carry signals related to the visual control of reaching movements (Inoue et al., 1998; Jouffrais and Boussaoud, 1999; Cisek and Kalaska, 2002) and the relationship between visual and proprioceptive information concerning the arm in space (Hoshi and Tanji, 2000; Lloyd et al., 2003). In addition, activity in this area has been shown to be correlated with the adaptation of reaching movements (Clower et al., 1996).

Transcranial magnetic stimulation (TMS) can be used to probe whether an area of the brain is necessary for normal task performance. It is thought to stimulate neural tissue underlying the skull site at which the coil is placed. This effect can be excitatory, as is the case when the motor cortex is stimulated and muscle activity results, or disruptive, as quite often occurs when subjects are attempting to perform a task that requires a distinct pattern of activity within the brain region being stimulated. The goal of the current study was to use TMS to examine the contribution of the PMd to prism adaptation specifically as it relates to the error correction and remapping processes. 


\section{Materials and Methods}

Participants. Five right-handed healthy subjects (three males; mean age, $25 \pm 3.5$ years) with no known neurological deficits affecting either vision or limb movements participated in the study. Before participating, the subjects were informed of the experimental procedures and provided written consent. The local human subjects committee had approved the procedures.

Experimental setup. The subject sat at a table looking down at a semisilvered mirror onto which target images were projected. The tip of the right index finger was instrumented with an infrared emitting diode that was monitored by a Watsmart system (sampled at $200 \mathrm{~Hz}$ ), a light-emitting diode (LED) that when illuminated provided visual feedback through the mirror regarding the position of the hand, and a small force transducer that signaled the end of the movement when contact was made with the tabletop. Right-handed pointing movements were made underneath the mirror from a home position $\sim 15 \mathrm{~cm}$ in front of the subject's midline to a target projected $20 \mathrm{~cm}$ anterior to the home position. Movement onset was signaled when an infrared beam positioned $1 \mathrm{~cm}$ anterior to the home position was interrupted by the initiation of the pointing response. After completion of each movement, the subject returned the hand to the home position as marked on the tabletop with a small disk.

Experimental procedure. The experiment consisted of preadaptation, adaptation, and postadaptation phases. During the preadaptation and postadaptation phases, the subject completed three separate tasks in a counterbalanced order. In the total shift test, the subject made 10 pointing movements without visual feedback from the hand to the target positioned straight ahead of the home position. This test measures the aftereffect of the adaptation phase. In the proprioceptive shift test, the subject pointed 10 times without vision of the hand or any target to a position that they perceived to be straight ahead. This tests for any changes in the felt position of the arm. In the visual shift test, a target appeared $30 \mathrm{~cm}$ to the left or right of straight ahead and started moving toward the center of the display at $10 \mathrm{~cm} / \mathrm{s}$. The subject was allowed to track the target motion with the eyes and was required to raise their right index finger when they perceived the target to be straight ahead. This tests for any changes in the visual perception of space.

During the adaptation phase, the subject donned 30 diopter prism goggles, which induced a shift in the visual field of $\sim 17^{\circ}$ to the right and completed 40 pointing movements with visual feedback provided by the illuminated LED on the index finger. In the full vision condition, the LED was illuminated from the onset of the movement, whereas in the terminal vision condition, the LED was illuminated for $500 \mathrm{~ms}$ only after the offset of the movement. In both conditions, the target was visible throughout the trial. Subjects were told to avoid using any explicit cognitive strategies such as pointing to the side of the perceived position of the target to achieve accuracy.

During the adaptation phase, a single pulse of transcranial magnetic stimulation (70 mm butterfly coil; Magstim, Whitland, UK) was applied at $110 \%$ of the motor threshold over the left premotor cortex on each trial. Before each session, the motor hot point was located over the left motor cortex and the motor threshold for eliciting twitches in the first dorsal interosseus muscle of the right hand was determined. The PMd site was then localized by moving the stimulating coil to a position $2 \mathrm{~cm}$ anterior and $1 \mathrm{~cm}$ medial to the motor hot point. This site is based on previous TMS studies in which the more dorsal aspect of the premotor cortex was targeted (Schluter et al., 1998; Munchau et al., 2002; Mochizuki et al., 2004). The coil was oriented at a $45^{\circ}$ angle to the midline with the handle pointing backwards. The lack of spread of the current toward the motor cortex was confirmed by the fact that finger twitches were no longer observed with the premotor cortex stimulation. Although we did not directly test it, we assumed based on the lack of spread to the motor cortex that the stimulation also did not spread to the more ventral aspects of the premotor cortex. The coil was held in place with a clamping system, and the head was stabilized with a chin rest.

We reasoned that if an area of the brain was engaged in the adaptation process in the full vision and terminal vision conditions, it would be most likely do so at the beginning and end of the movement, respectively. Therefore, in separate sessions, TMS was applied during the adaptation phase either at the onset of every movement (i.e., when the infrared beam was interrupted) in the full vision condition or at the end of every movement (i.e., when the force transducer registered the impact of the index finger with the table) in the terminal vision condition. Four of the subjects also completed the converse combination of conditions as controls (i.e., full vision with stimulation at the end of the movement and terminal vision with stimulation at movement onset). In addition, the terminal vision and full vision conditions were also performed without TMS. Each subject completed the different combination of conditions in a different order with at least $2 \mathrm{~d}$ rest in between.

Data analysis. Performance change during the prism exposure period was quantified by measuring changes in movement error and displacement/distance ratio across trials. Movement error was defined as the mediolateral distance between the target and the final position of the index finger. Errors to the left were considered negative, whereas those to the right were positive. We approximated the rate of adaptation by fitting the error scores across the adaptation trials with an exponential function and calculating the decay constant (Martin et al., 1996; Morton and Bastian, 2004). The displacement/distance ratio was used as an index of the degree to which on-line error corrections occurred. It was calculated as the ratio between the total reaching displacement and the distance between the starting and ending position of the index finger. Thus, a perfectly straight trajectory would yield a ratio score of one. In addition to these adaptation period measures, the changes induced by the prism adaptation were monitored by calculating the difference in the total, proprioceptive, and visual shift tests between the preadaptation and postadaptation periods. Statistical analyses were completed separately for the full vision and terminal vision conditions using repeated-measures ANOVAs with stimulation presence/time as a within-subjects factor.

\section{Results}

\section{Full vision condition: adaptation phase}

The clearest evidence that adaptation took place while the subject wore prism goggles is the change in movement error. Figure $1 \mathrm{~A}$ displays typical hand paths from an individual subject during early, middle, and late trials within the adaptation phase. Trials without TMS are displayed on the left and those with TMS at movement onset are shown on the right. In both cases, the size of the error at the end of the movement decreases as the adaptation phase progresses, especially during the initial trials. Figure $1 B$ plots group averages for the error scores across the 40 movements made during the adaptation phase in the full vision condition. The black circles represent the no TMS condition, the dark gray triangles the condition in which TMS was delivered at movement offset, and the gray circles the condition in which TMS was delivered at movement onset. In each case, the initial trials displayed large errors in the direction induced by the prism goggles, which were subsequently reduced to near zero (thick horizontal line) with repeated movements toward the target. The rate at which adaptation occurred was calculated by fitting an exponential decay function to the data for each subject. This analysis revealed that the adaptation rate was significantly slower when TMS was delivered at movement onset than when it was delivered at movement offset or not at all $\left(F_{(2,13)}=3.25 ; p<0.05\right)$ (Fig. $\left.1 C\right)$. This resulted in a greater number of trials before the movement error returned close to zero. We suggest that this difference implies that subjects complied with our request to avoid using an explicit cognitive strategy to achieve accuracy; if they would have done so, their error scores would have followed a similar time course to that observed in the other conditions. Thus, TMS over the PMd appears to disrupt the ability to adapt to the visual perturbation induced by prism goggles when it is delivered at the onset of each trial in which the subject can see their hand throughout the movement.

The slower adaptation rate when TMS was delivered at movement onset presumably reflects a disruption in the ability to use visual feedback to make corrections under these circumstances. 
A
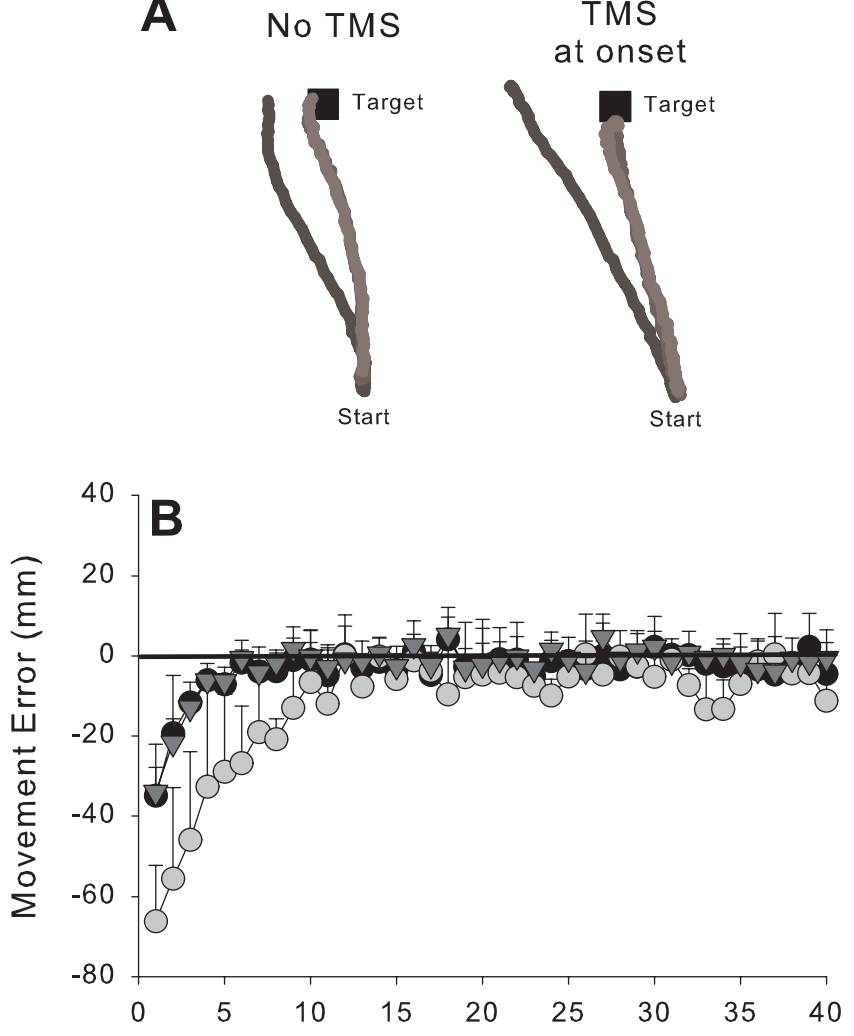

Adaptation Trial Number
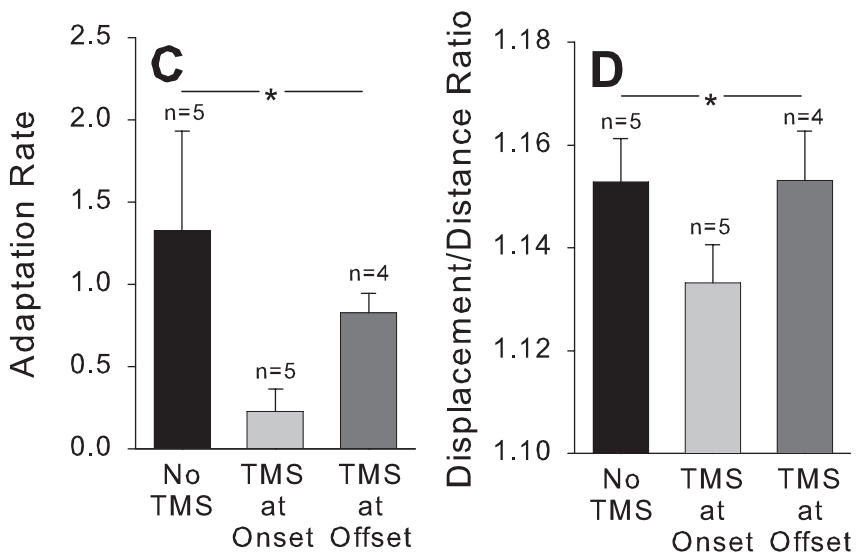

Figure 1. Full vision condition. $\boldsymbol{A}$, Typical hand paths from an individual subject in the early (black), middle (dark gray), and late (light gray) portions of the adaptation period without TMS (left) and with TMS given at movement onset (right). $\boldsymbol{B}$, Group average for movement error plotted as a function of adaptation trial number in trials without TMS (filled circles), trials with TMS delivered at movement onset (light gray circles), and trials with TMS delivered at movement offset (dark gray triangles). C, Group average for adaptation rate in the trials without TMS (black bar), with TMS delivered at movement onset (light gray bar), or at movement offset (dark gray bar). D, Group average for displacement/distance ratio in the trials without TMS (black bar), with TMS delivered at movement onset (light gray bar), or at movement offset (dark gray bar). The asterisks indicate significant difference across conditions. Error bars represent 1 intersubject SE.

Examination of the example hand paths (Fig. $1 \mathrm{~A}$ ) suggests that this is indeed the case: the hand paths tend to be straighter when TMS is given at movement onset, especially during the earliest trials in the adaptation phase. If this is the case, then it should be reflected in the displacement/distance ratio scores because these represent the degree to which on-line error corrections occurred. Analysis of the displacement/distance ratio scores during the first 10 trials of the
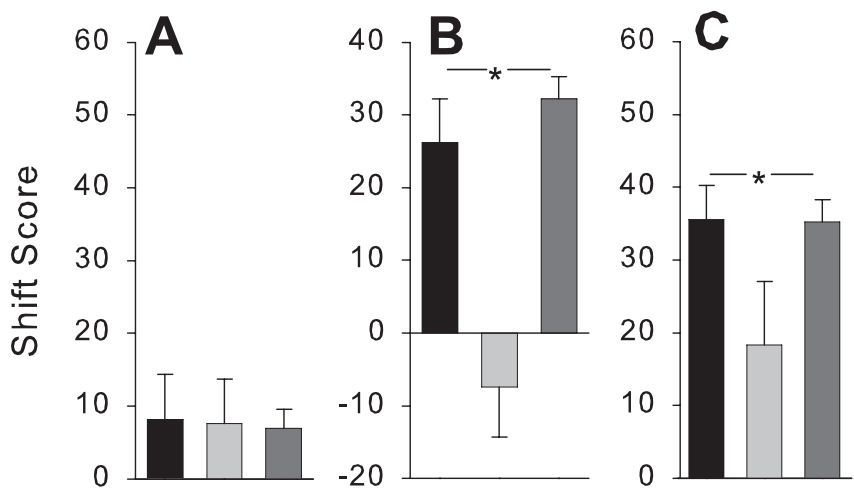

Figure 2. Full vision condition. Group averages for the changes induced in the visual $(\boldsymbol{A})$, proprioceptive $(\boldsymbol{B})$, and total $(\boldsymbol{C})$ shift tests as a result of the adaptation trials when TMS was not given $(n=5)$ (black bars), delivered at movement onset $(n=5)$ (light gray bars), or delivered at movement offset $(n=4)$ (dark gray bars) are shown. The asterisks indicate significant difference across conditions. Error bars represent 1 intersubject SE.

adaptation phase showed that it was significantly smaller when TMS was delivered at movement onset than when it was delivered at movement offset or not at all $\left(F_{(2,13)}=2.14 ; p<0.05\right)($ Fig. $1 D)$. In other words, the movement trajectories were straighter and less accurate when the PMd was disrupted at the beginning of the movement, reflecting the fact that on-line corrections were required to counteract the influence of the prism goggles.

\section{Full vision condition: preadaptation and postadaptation phase}

The shift test scores provided an index of how much and in what way the exposure to the prism goggles affected sensorimotor control processes. We calculated the change induced by the adaptation phase in each shift test score with respect to the performance of each subject before adaptation. Figure $2 A-C$ displays the group means for the difference in the visual, proprioceptive, and total shift scores, respectively, from the preadaptation to the postadaptation phase. ANOVAs were used to compare the magnitude of these difference scores across the different conditions. This demonstrated that TMS delivered at the onset of the movement during the adaptation phase caused a significant reduction in the total $\left(F_{(2,13)}=3.34 ; p<0.05\right)$ and proprioceptive shift $\left(F_{(2,13)}=6.19 ; p<0.05\right)$ scores but not the visual shift $\left(F_{(2,13)}=\right.$ $0.56 ; p>0.05)$ score. Thus, disruption of the PMd activity at the onset of movement during the adaptation phase caused a reduction in the size of the aftereffect (i.e., the total shift score). This was accompanied by a markedly smaller change in the felt position of the arm (i.e., the proprioceptive shift score) resulting from the adaptation. In contrast, the more subtle change induced by the adaptation in the visual perception of straight ahead (i.e., the visual shift score) was not affected by TMS delivered at movement onset over the premotor cortex.

\section{Terminal vision condition: adaptation phase}

Figure $3 A$ plots the average error scores for the group across the adaptation phase in the terminal vision condition. Unlike in the full vision condition, TMS did not affect the rate of adaptation in this condition regardless of whether it was given at movement onset or offset $\left(F_{(2,13)}=0.95 ; p>0.05\right)$ (Fig. 3B). As expected, the presence of on-line corrections, as inferred from the displacement/distance ratio, was also uninfluenced by TMS delivered under these conditions. Thus, when visual feedback concerning pointing accuracy was only available at the end of the movement, disruption of PMd activity did not affect the adaptation process. 

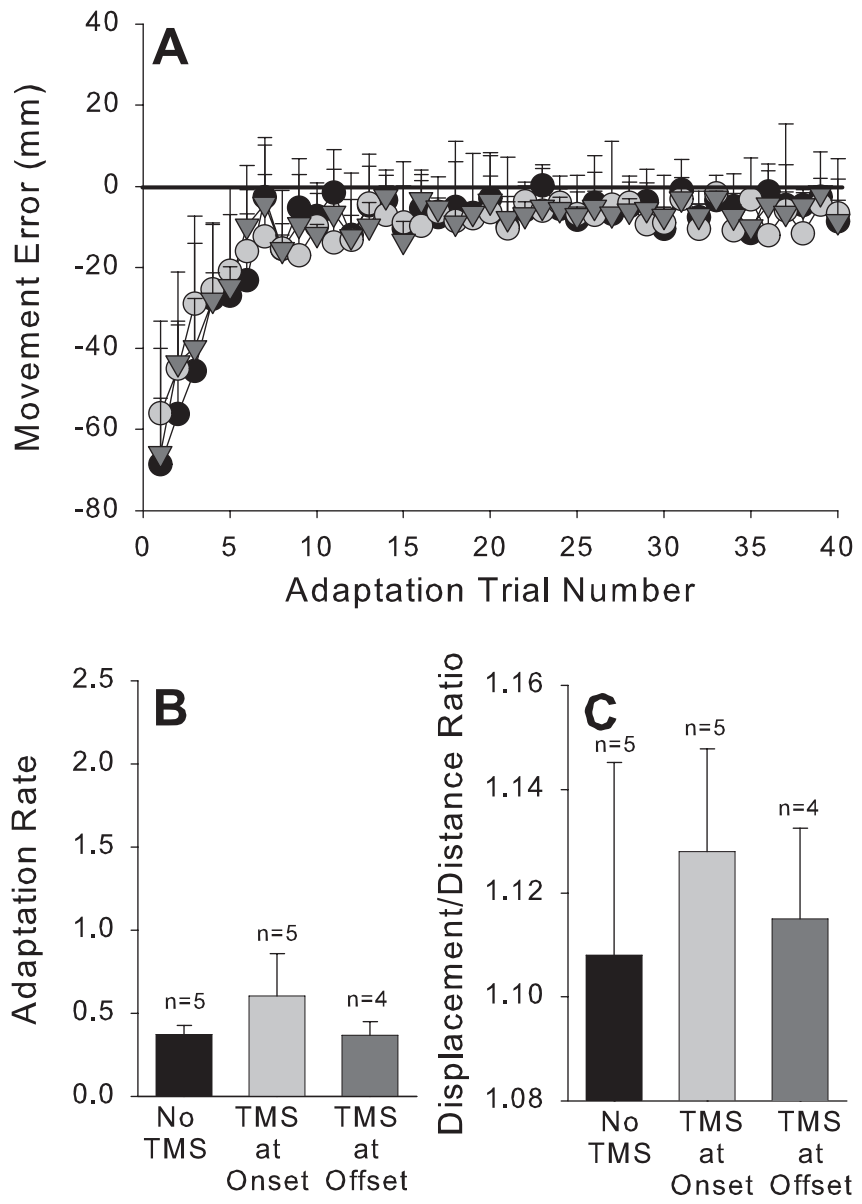

Figure 3. Terminal vision condition. Figure conventions as in Figure 1.
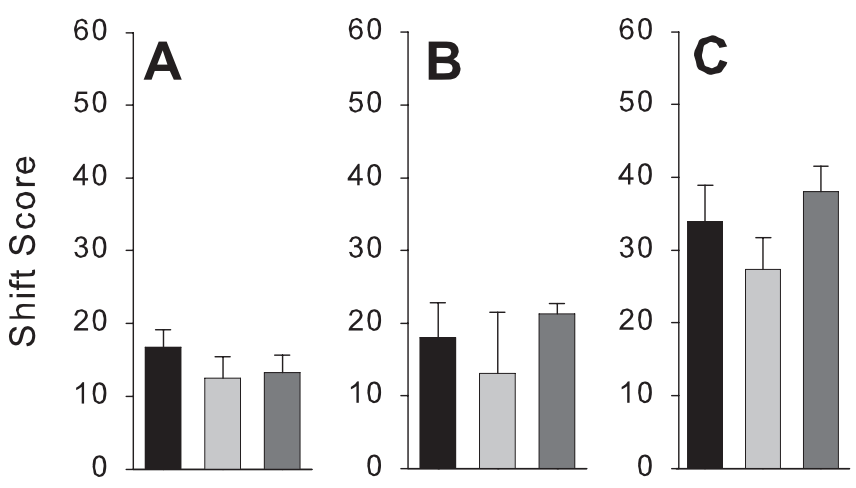

Figure 4. Terminal vision condition. Figure conventions as in Figure 2.

\section{Terminal vision condition: preadaptation and} postadaptation phase

Figure $4 A-C$ displays the group means for the difference in the visual, proprioceptive, and total shift scores from the preadaptation to the postadaptation phase in the terminal vision condition. In each case, there was no difference in the change in the shift score as a result of TMS being delivered to the premotor cortex either at movement onset or movement offset (visual shift: $F_{(2,13)}=0.63, p>0.05$; proprioceptive shift: $F_{(2,13)}=0.29, p>0.05$; total shift: $F_{(2,13)}=0.51$, $p>0.05)$. Thus, in addition to the lack of influence on the adaptation phase itself, TMS at movement onset or offset also did not affect the magnitude of the aftereffect, the changes in the felt position of the arm, or the changes in the visual perception of space that were induced by wearing the prism goggles in the terminal vision condition.

\section{Discussion}

The goal of this experiment was to gain a better understanding of the contribution of the human PMd to the error correction and remapping processes underlying sensorimotor adaptation. We addressed this question by interfering with the normal function of the PMd using single pulses of TMS delivered either at movement onset or offset during exposure to prism goggles. We found that when vision of the hand was available throughout the response, TMS delivered at movement onset slowed down the initial rate of adaptation such that it took more trials before the pointing responses became accurate. These initially inaccurate trials were the result of straighter movement trajectories, implying that the TMS disrupted the ability to make on-line error corrections to compensate for the visual perturbation induced by the goggles. Moreover, the slower rate of adaptation led to a smaller aftereffect (total shift score), which was accompanied by a smaller change in the felt position of the arm (proprioceptive shift score). Thus, we conclude that the PMd normally plays a role in sensorimotor adaptation under these conditions by contributing to the on-line error corrections that are required to reacquire the target. One of the consequences of this correction process is that over the course of a block of trials, a remapping of the felt position of the arm occurs. Clearly, this error correction process and its consequences were disrupted by TMS over the PMd. However, these effects were specific to the condition in which full vision of the hand was available and TMS was given at movement onset: TMS given at movement offset with full vision of the hand or at either movement onset or offset when vision of the hand was available only at the end of the response did not affect the adaptation process.

Previous research provided evidence that is consistent with the role of the PMd in sensorimotor adaptation. Cells in the PMd code the relationship between visual objects in the field of view and somatosensory input originating from the arm (Hoshi and Tanji, 2000; Lloyd et al., 2003). Consistent with this multisensory processing, activity in the premotor cortex also contributes to the visual control of reaching movements (Inoue et al., 1998; Jouffrais and Boussaoud, 1999; Cisek and Kalaska, 2002) and is modulated during a variety of sensorimotor learning tasks (Clower et al., 1996; Shadmehr and Holcomb, 1997; Brasted and Wise, 2004). Clower and colleagues (1996) specifically examined the brain areas involved in prism adaptation and showed that although the premotor cortex played a role in the error correction component of adaptation, it did not explicitly contribute to the remapping that is thought to be responsible for the adaptation itself. Instead, they found that remapping-related activation was restricted to the posterior parietal cortex (PPC). Our results confirm those of the Clower study and further suggest that the PMd is necessary for the on-line error corrections that underlie the remapping of the felt position of the arm during sensorimotor adaptation. This supportive role of the PMd is most likely performed via reciprocal projections (Battaglia-Mayer et al., 2003) with the PPC.

Desmurget and colleagues (1999) showed previously that TMS over the PPC also disrupts on-line error corrections during reaching response to targets that suddenly change position at movement onset. Although this is somewhat different from the situation with prism goggles in that subjects did not know from one trial to the next whether the target would move or, if it did, which direction it would go, it is similar enough to suggest that both the PPC and PMC contribute to on-line error corrections and that the PPC also is directly involved in remapping of pro- 
prioceptive signals onto visual representations (Clower et al., 1996). Indeed, Della-Maggiore and colleagues (2004) have shown adaptation itself is markedly disrupted when TMS is delivered over the PPC during reaching movements performed in a novel force field. Furthermore, a recent functional magnetic resonance imaging study by Diedrichsen and coworkers demonstrated PPC activation related to kinematic, but not dynamic, execution errors during adaptive reaching responses (Diedrichsen et al., 2005).

Imamizu et al. (2000) suggested that sensorimotor learning occurs as a result of error feedback from one trial being used by the subject to improve performance on the subsequent trial. By this means, accuracy increases as the subject progresses through the training trials. Based on previous behavioral evidence (Redding and Wallace, 1988, 1996, 1997, 2000), we suggested that trial-to-trial corrections are more likely to account for the learning that occurs in the terminal vision condition, whereas, it is less likely to contribute to the full vision learning. With this in mind, the current results are consistent with the idea that the premotor cortex does not make a substantial contribution to trial-to-trial learning; if it did, then TMS over the premotor cortex would slow adaptation and reduce the size of the aftereffects in the terminal vision as well as the full vision condition. Rather, the cerebellum appears to play a key role in this form of learning: neural activity in the cerebellum has been shown to be related to errors within a reaching movement (Kitazawa et al., 1998) and the reduction in these errors across trials (Imamizu et al., 2000), and cerebellar damage disrupts prism adaptation during throwing responses when on-line error corrections are impossible (Martin et al., 1996).

Finally, although we targeted the PMd, the ventral aspect of the premotor area (PMv) also contributes to the visual control of reaching in that it encodes the mapping between visual and proprioceptive information concerning the arm in space (Ehrsson et al., 2004; Graziano, 1999). The PMv has also been shown to contribute to the adaptation of reaching responses (Kurata and Hoshi, 1999; Inoue et al., 2000; Krakauer et al., 2004). Hoshi and Tanji (2004) suggested that the PMv is primarily involved in integrating visuospatial signals specifying the goal of the reaching response, whereas the PMd is more involved in mapping signals related to the arm to be used for the reaching response and the target to be reached. Together, this is broadly consistent with the current finding that TMS delivered over PMd disrupts on-line corrections during prism adaptation.

\section{Conclusions}

We demonstrated that the human PMd normally contributes to sensorimotor adaptation induced by wearing prism goggles. However, it does so only under a restricted set of circumstances, in particular, during the early portions of the movement when vision of the hand is available. During this time, the discrepancy between the expected trajectory of the hand and the perturbed visual feedback is normally used to make on-line adjustments to the movement. Our results demonstrate that the PMd is necessary for this process. When it is disrupted with TMS, the trajectory modifications are reduced and adaptation suffers as a result.

\section{References}

Battaglia-Mayer A, Caminiti R, Lacquaniti F, Zago M (2003) Multiple levels of representation of reaching in the parieto-frontal network. Cereb Cortex 13:1009-1022.

Brasted PJ, Wise SP (2004) Comparison of learning-related neuronal activity in the dorsal premotor cortex and striatum. Eur J Neurosci 19:721-740.

Cisek P, Kalaska JF (2002) Modest gaze-related discharge modulation in monkey dorsal premotor cortex during a reaching task performed with free fixation. J Neurophysiol 88:1064-1072.

Clower DM, Hoffman JM, Votaw JR, Faber TL, Woods RP, Alexander GE (1996) Role of posterior parietal cortex in the recalibration of visually guided reaching. Nature 383:618-621.

Della-Maggiore V, Malfait N, Ostry DJ, Paus T (2004) Stimulation of the posterior parietal cortex interferes with arm trajectory adjustments during the learning of new dynamics. J Neurosci 24:9971-9976.

Desmurget M, Epstein CM, Turner RS, Prablanc C, Alexander GE, Grafton ST (1999) Role of the posterior parietal cortex in updating reaching movements to a visual target. Nat Neurosci 2:563-567.

Diedrichsen J, Hashambhoy Y, Rane T, Shadmehr R (2005) Neural correlates of reach errors. J Neurosci 25:9919-9931.

Ehrsson HH, Spence C, Passingham RE (2004) That's my hand! Activity in premotor cortex reflects feeling of ownership of a limb. Science 305:875-877.

Graziano MS (1999) Where is my arm? The relative role of vision and proprioception in the neuronal representation of limb position. Proc Natl Acad Sci USA 96:10418-10421.

Hoshi E, Tanji J (2000) Integration of target and body-part information in the premotor cortex when planning action. Nature 408:466-470.

Hoshi E, Tanji J (2004) Functional specialization in dorsal and ventral premotor areas. Prog Brain Res 143:507-511.

Imamizu H, Miyauchi S, Tamada T, Sasaki Y, Takino R, Putz B, Yoshioka T, Kawato M (2000) Human cerebellar activity reflecting an acquired internal model of a new tool. Nature 403:192-195.

Inoue K, Kawashima R, Satoh K, Kinomura S, Goto R, Koyama M, Sugiura M, Ito M, Fukuda H (1998) PET study of pointing with visual feedback of moving hands. J Neurophysiol 79:117-125.

Inoue K, Kawashima R, Satoh K, Kinomura S, Sugiura M, Goto R, Ito M, Fukuda H (2000) A PET study of visuomotor learning under optical rotation. NeuroImage 11:505-516.

Jouffrais C, Boussaoud D (1999) Neuronal activity related to eye-hand coordination in the primate premotor cortex. Exp Brain Res 128:205-209.

Kitazawa S, Kimura T, Yin PB (1998) Cerebellar complex spikes encode both destinations and errors in arm movements. Nature 392:494-497.

Krakauer JW, Ghilardi MF, Mentis M, Barnes A, Veytsman M, Eidelberg D, Ghez C (2004) Differential cortical and subcortical activations in learning rotations and gains for reaching: a PET study. J Neurophysiol 91:924-933.

Kurata K, Hoshi E (1999) Reacquisition deficits in prism adaptation after muscimol microinjection into the ventral premotor cortex of monkeys. J Neurophysiol 81:1927-1938.

Lloyd DM, Shore DI, Spence C, Calvert GA (2003) Multisensory representation of limb position in human premotor cortex. Nat Neurosci 6:17-18.

Martin TA, Keating JG, Goodkin HP, Bastian AJ, Thach WT (1996) Throwing while looking through prisms. I. Focal olivocerebellar lesions impair adaptation. Brain 119:1183-1198.

Mochizuki H, Huang YZ, Rothwell JC (2004) Interhemispheric interaction between human dorsal premotor and contralateral primary motor cortex. J Physiol (Lond) 561:331-338.

Morton SM, Bastian AJ (2004) Prism adaptation during walking generalizes to reaching and requires the cerebellum. J Neurophysiol 92:2497-2509.

Munchau A, Bloem BR, Irlbacher K, Trimble MR, Rothwell JC (2002) Functional connectivity of human premotor and motor cortex explored with repetitive transcranial magnetic stimulation. J Neurosci 22:554-561.

Redding GM, Wallace B (1988) Components of prism adaptation in terminal and concurrent exposure: organization of the eye-hand coordination loop. Percept Psychophys 44:59-68.

Redding GM, Wallace B (1996) Adaptive spatial alignment and strategic perceptual-motor control. J Exp Psychol Hum Percept Perform 22:379-394.

Redding GM, Wallace B (1997) Prism adaptation during target pointing from visible and nonvisible starting locations. J Mot Behav 29:119-130.

Redding GM, Wallace B (2000) Prism exposure aftereffects and direct effects for different movement and feedback times. J Mot Behav 32:83-99.

Schluter ND, Rushworth MF, Passingham RE, Mills KR (1998) Temporary interference in human lateral premotor cortex suggests dominance for the selection of movements. A study using transcranial magnetic stimulation. Brain 121:785-799.

Shadmehr R, Holcomb HH (1997) Neural correlates of motor memory consolidation. Science 277:821-825. 